\title{
ON SUBBAND MOBILITIES OBSERVED IN $\delta$-DOPED AlGaAs/GaAs QUANTUM WELLS AND GaAs LAYERS*
}

\author{
L. DobaCZewski ${ }^{a}$, D.K. MAUdE ${ }^{b}$, M. Missous ${ }^{c}$ AND J.C. PoRTaL ${ }^{b}$ \\ ${ }^{a}$ Institute of Physics, Polish Academy of Sciences \\ Al. Lotników 32/46, 02-668 Warszawa, Poland

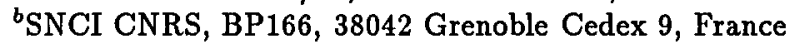 \\ ${ }^{c}$ Department of Electrical Engineering and Electronics, UMIST \\ P.O. Box 88, Manchester M60 1QD, Great Britain
}

Electronic transport phenomena in molecular beam epitaxy grown silicon $\delta$-doped $\mathrm{AlGaAs} / \mathrm{GaAs}$ quantum wells and $\mathrm{GaAs}$ layers were investigated. Observations of the Shubnikov-de Haas oscillations allowed to deduce the redistribution of electrons among energy subbands formed by V-shaped and rectangular wells for GaAs layers and the $\mathrm{AlGaAs} / \mathrm{GaAs}$ quantum wells, respectively. In both cases the effects of illumination upon individual subband mobilities and carrier concentrations were studied and the manifestation of the DX centres was demonstrated.

PACS numbers: 71.25.Hc, 71.55.Eq, 73.20.Dx

It has already been demonstrated that measurements of the Shubnikovde Haas (SdH) oscillations for two-dimensional electron gas (2DEG) systems give concentrations and mobilities of electrons in individual energy subbands. This is usually achieved when these oscillations (as a function of reciprocal magnetic field) are spectrally analyzed by a fast Fourier transform (FFT) technique. Positions of the FFT peaks on the electron sheet concentration scale indicate the occupations for each energy subband, and the peak broadening is a measure of the carrier mobility for the given subband.

There are numerous experimental results demonstrating that the donor doping process of III-V semiconductors creates localized energy states (the DX centres). They are resonant with the conduction band in GaAs at ambient pressure and emerge in the band gap for higher hydrostatic pressures. For a very heavily doped GaAs the Fermi level can approach the DX energy level resulting in its partial occupation. This heavy doping regime can be realized locally by planar $(\delta)$

*This work was financially supported in part by the grant No. 2 P302 11705 of the State Committee for Scientific Research (Republic of Poland), Science and Engineering Research Council grants in UK, and Ministère des Affaires Etrangères in France. 
doping process and the DX states in such regions have already been observed [1]. The presence of the DX centres causes the metastability effects in the crystal, and consequently, can affect the transport characteristics of $\delta$-doped structures. The results of observations of metastable occupation and electron mobility in the energy subbands formed in two-dimensional structures are reported.

In this study we have investigated the electronic transport phenomena in silicon $\delta$-doped AlGaAs/GaAs quantum wells and GaAs layers. The samples were grown by molecular beam epitaxy with different sheet concentration of donors for the case of GaAs layers. The AlGaAs/GaAs quantum wells were moderately doped $\left(n_{\mathrm{s}}=2 \times 10^{12} \mathrm{~cm}^{-2}\right.$ ) and the plane of dopants was positioned in the middle of the well. The Shubnikov-de Haas measurements were performed to investigate the redistribution of electrons among subbands formed by $\mathrm{V}$-shaped and rectangular wells for GaAs layers and the AlGaAs/GaAs quantum wells, respectively. In both cases the effects of illumination upon individual subband mobilities and carrier concentrations were studied.

For the case of the highly doped GaAs, where the peak 2D electron concentration approaches $1 \times 10^{13} \mathrm{~cm}^{-2}$, novel changes in the subband transport characteristics were observed, not seen in the samples with the lower doping level. We attribute these effects to the presence of DX centre phenomena. When the sample was cooled down to $4.2 \mathrm{~K}$ in darkness three occupied subbands of the V-shaped well could be observed. The FFT analysis of the SdH oscillations revealed that the mobilities equaled 800,2700 , and $1800 \mathrm{~cm}^{2} /(\mathrm{V} \mathrm{s})$ for the subband index $i=0$, 1 , and 2 , respectively. The sample illumination with the above band gap light substantially and metastably modified not only the electron concentrations for the subbands but the mobilities as well. After the illumination the mobilities were found to be equal to 1700,2400 , and $3400 \mathrm{~cm}^{2} /(\mathrm{V} \mathrm{s})$ for $i=0,1$, and 2 , respectively. The metastability of this effect clearly indicated that the additional electrons, which changed the redistribution of charge among the subbands, originated from the DX centres. However, the relative change of the mobilities could not be explained neither by an increase in uncompensated charge of ionized donors nor a destruction of a spatial correlation among donors.

This variations in the individual subband transport properties was explained as being due to a shift of the electronic wave function confined in the potential well. The above band gap illumination creates electron-hole pairs in the whole layer of GaAs. Only in the region between the sheet of donors and a substrate the number of the minority carriers can be non-negligible because on the surface side of the $\delta$-plane the surface recombination enormously reduces the hole lifetime. As it was demonstrated by us [2] the DX centres, as acceptors, when occupied have long-range Coulomb potential attractive for holes. The holes can approach the DX centres only from one side and, due to the one-side electron-hole recombination process, cause a large asymmetry of the potential well. As a result, for the subbands (even ones) where the electronic wave function has a maximum at the well centre (at the scattering defects) the appearance of the well asymmetry should be accompanied with an increase in the mobility, and for the odd subbands the effect should be opposite. This is exactly what is observed in the experiment (see Ref. [3] for details). 
For the $\delta$-doped AlGaAs/GaAs quantum well only one electron subband was observed. Due to the fact that the electron wave function is in this case more confined by the well walls it overlaps much more with the sheet of the charged centres. This results in a substantially lower mobility for this subband $\left(320 \mathrm{~cm}^{2} /(\mathrm{V} \mathrm{s})\right)$ than it was for the GaAs layer. The illumination of the sample at low temperature with the below band gap (of GaAs) light causes only a small change in the mobility (to $340 \mathrm{~cm}^{2} /(\mathrm{V} \mathrm{s})$ ) but increases substantially the concentration (change from $5.5 \times 10^{11}$ to $8.4 \times 10^{11} \mathrm{~cm}^{-2}$ ). This persistent effect can be explained as being due to metastable and homogeneous photoionization of the DX centres with no change of the well symmetry. For the GaAs sample with the same planar doping level no metastability phenomena were observed. However, the rectangular quantum well more effectively confines the electronic wave function than it is in the V-shaped well for the first subband, thus this subband can approach the resonant DX state for lower doping level than it happened for the V-shaped well in GaAs.

When for illumination the above band gap light is used there is a much larger change in the mobility (to $500 \mathrm{~cm}^{2} /(\mathrm{V} \mathrm{s})$ ) accompanied by further but less dramatic increase in the sheet electron concentration (to $10 \times 10^{11} \mathrm{~cm}^{-2}$ ). This increase in the electron mobility can be again explained by the effective well asymmetrization due to the electron-hole recombination related to the presence of the DX states in the well, similarly to the case of the $\delta$-doped GaAs samples.

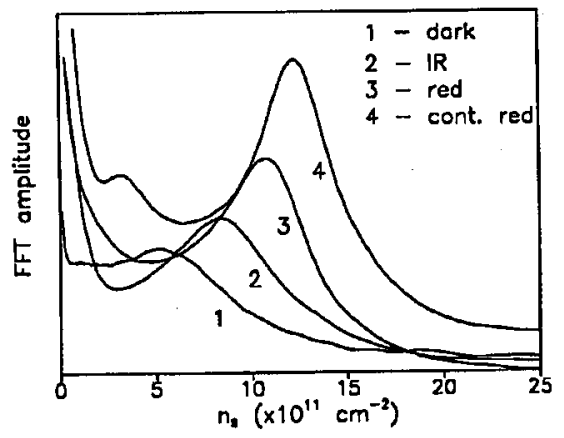

Fig. 1. The FFT spectra of the SdH oscillations for the $\delta$-doped AlGaAs/GaAs quantum well measured at four experimental conditions: 1 - in darkness, 2 - after below band gap illumination, 3 - after above band gap illumination, and 4 - during intensive above band gap illumination. In the latter case the appearance of the second subband is seen.

In Fig. 1 there are the FFT spectra of the SdH oscillations for the $\delta$-doped $\mathrm{AlGaAs} / \mathrm{GaAs}$ quantum well. The spectra were measured at four experimental conditions: in darkness, after below band gap illumination, after above band gap illumination, and during intensive above band gap illumination. The increase in the sheet electron concentration is clearly seen as a shift of the peak position. In this type of presentation the mobility for the given subband is proportional to the peak half width at half maximum (see e.g. Ref. [4] for details). 


\section{References}

[1] S. Arscott, M. Missous, L. Dobaczewski, Semicond. Sci. Technol. 7, 620 (1992).

[2] L. Dobaczewski, P. Kaczor, Z.R. Żytkiewicz, M. Missous, F. Saleemi, P. Dawson, A.R. Peaker, J. Appl. Phys. 72, 3198 (1992).

[3] S. Arscott, M. Missous, L. Dobaczewski, P.C. Harness, D.K. Maude, J.C. Portal, in: Semiconductor Heterostructures for Photonic and Electronic Applications, Eds. C.W. Tu, D.C. Houghton, R.T. Taung, Materials Research Society Symposium Proceedings, Vol. 281, Materials Research Society, Pittsburgh 1993, p. 19.

[4] S. Yamada, T. Makimoto, Appl. Phys. Lett. 57, 1022 (1990). 